\title{
Minor Postoperative Complications Related to Anesthesia in Elective Gynecological and Orthopedic Surgical Patients at a Teaching Hospital in Kingston, Jamaica
}

\author{
Ingrid Tennant 1, Richard Augier 2, Annette Crawford-Sykes ${ }^{1}$, Doreen Ferron-Boothe ${ }^{3}$, Nicola Meeks-Aitken ${ }^{3}$, \\ Karen Jones ${ }^{3}$, Georgiana Gordon-Strachan ${ }^{4}$, Hyacinth Harding-Goldson 5
}

\begin{abstract}
Summary: Tennant I, Augier R, Crawford-Sykes A, Ferron-Boothe D, Meeks-Aitken N, Jones K, Gordon-Strachan G, Harding-Goldson H - Minor Postoperative Complications Related to Anesthesia in Elective Gynecological and Orthopedic Surgical Patients at a Teaching Hospital in Kingston, Jamaica.
\end{abstract}

Background and objectives: Minor postoperative anesthetic complications may increase patient discomfort and dissatisfaction and delay recovery. This paper sought to determine the frequency of minor complications in the first 48 hours postoperatively reported by elective gynecological and orthopedic surgical patients at the University Hospital of the West Indies, Jamaica. Overall satisfaction with anesthetic care and possible risk factors for developing complications were also assessed.

Methods: A prospective, descriptive cohort study was undertaken with patient interviews 24 to 48 hours after anesthesia. Data were analyzed using SPSS version 12 and assessed using the $\chi^{2}$-square test and multiple logistic regression models.

Results: Five hundred and five (505) patients were included, with 374 females (74\%). Most were ASA I (55\%) or ASA II (38\%) and had general anesthesia (80\%). A total of $419(83 \%)$ patients reported at least one complication postoperatively. The most frequently reported complications were sore throat (44\%), nausea (30\%), vomiting (24\%), and thrombophlebitis (20\%). The mode of the Verbal Numerical Rating Score (VNRS) for each complication ranged between 2 and 5, suggesting that most did not cause severe distress. Age less than 45 years (OR 2.22, 95\% $\mathrm{Cl} 1.34-$ $3.69, \mathrm{p}=0.002$ ) and female gender (OR 3.64, 95\% Cl 2.14-6.20, $\mathrm{p}<0.001$ ) were identified as significant independent variables. Most patients regarded their anesthetic experience as excellent $(51 \%)$ or very good $(22 \%)$.

Conclusion: This study showed a comparatively high incidence of minor postoperative complications (83\%), but low reported severity of symptoms and a high overall satisfaction rate. Special attention should be paid to reduce these minor complications through more meticulous anesthetic technique.

Keywords: Anesthesia Department; Patient Satisfaction; Postoperative Complications; Postoperative Period.

(C2012 Elsevier Editora Ltda. All rights reserved.

\section{INTRODUCTION}

Postoperative complications related to anesthesia cover a wide spectrum of severity from mildly distressing with no longterm sequelae to death or permanent disability. It has been suggested that several factors contribute to postoperative morbidity and length of hospital stay, including comorbidities

\footnotetext{
Received from University of the West Indies, Jamaica.

1. MB. BS, DM, Lecturer; Consultant Anesthesiologist, University of the West Indies 2. MB.BS, DM; Associate Lecturer; Consultant Anesthesiologist, University of the West Indies

3. Register Nurse; Research Nurse, University of the West Indies

4. PhD; Biostatistician; Faculty of Medical Sciences, University of the West Indies

5. MB.BS, DM; Senior Lecturer; Consultant Anesthesiologist, University of the West Indies

Submitted on Mat 7, 2011.

Approved on June 19, 2011.

Correspondence to:

Ingrid Tennant, MD

Department of Surgery Radiology, Anesthesia and Intensive Care

University of the West Indies

Kingston 7. Mona, Jamaica

E-mail: ingrid@ac-martin.com
}

and their pre-operative control, the surgical stress response, postoperative organ dysfunction, pain, poor nutrition and sleep disturbances ${ }^{1}$. Anesthetic technique and medications used may also contribute to postoperative complications. Common complaints include nausea and vomiting, surgical pain, sore throat, headache, drowsiness and dizziness, dental damage, peripheral nerve injury, and superficial thrombosis ${ }^{2}$. Awareness during anesthesia is a very rare but potentially devastating complication of anesthesia ${ }^{3}$.

The development of safer anesthetic agents, modes of delivery and improvements in patient monitoring and pain management over the past few decades have translated into a reduction in anesthetic risk. However, although there has been a decline in mortality and major morbidity rates, the incidences of minor and more common complications have not changed significantly ${ }^{2}$. These complications show a strong correlation with overall patient satisfaction with their anesthetic experience ${ }^{4}$, and may result in enough patient discomfort to justify changes in practice. Therefore, it is important for anesthesiologists to monitor clinical outcomes and use the information gained to improve quality of care 5 . 
Anesthetic complications intraoperatively and in the immediate postoperative period (recovery room) at the University Hospital of the West Indies (UHWI) have been documented ${ }^{6}$. This paper looked at minor complications reported by gynecological and orthopedic patients after elective surgery in the first 48 hour period post recovery from anesthesia and their overall satisfaction with anesthesia care.

\section{METHODS}

A prospective, descriptive cohort study was undertaken at the University Hospital of the West Indies (UHWI), Kingston, Jamaica. This is a 500 -bed, multidisciplinary, tertiary referral centre and teaching hospital affiliated to the University of the West Indies (UWI). Ethical approval was obtained from the UWI Faculty of Medical Sciences Ethics Committee. Elective orthopedic and gynecological patients receiving either a general or regional anesthetic were included. The following groups of patients were excluded: 1) Patients under 16 years of age; 2) Patients with a diagnosis of mental retardation or senile dementia; 3) Patients with hearing impairment; 4) Daycase patients (discharged within 24 hours post anesthesia); 5) Patients who refused or were unable to participate.

Sample size calculations (using Epi Info v. 3.3.2) indicated that a sample population of 486 was required to yield a power of $80 \%$ and a confidence interval of $95 \%$. This calculation was based on an estimated worst expected complications frequency of $10 \%$.

Data collected included the patient's age, gender, American Society of Anesthesiologists (ASA) physical status score, preoperative medical conditions, anesthetic technique, and length of anesthesia. Surgical procedures were graded according to perceived associated risk into major (such as Wertheim's hysterectomies, total knee replacements), intermediate (hysterectomies, open reduction and internal fixation of fractures), and minor (biopsies, or incision and drainage of abscesses) surgeries. Other information recorded included any intraoperative or recovery period complications, obtained from the anesthetic charts. Postoperative complications were documented via an interview and review of in-patient charts (24 to 48 hours after anesthesia) by trained research nurses or one of the research anesthesiologists. Defined criteria were used for each complication ${ }^{7}$. Patients were also asked to score the severity of the symptoms on a verbal numerical rating scale (VNRS) of zero to 10, where zero represented "not present" and 10 represented "the worst severity one could imagine". The level of patient satisfaction with their anesthesia care was assessed by two questions at the postoperative interview: "How would you rate your anesthetic?" (using a 7-point Likert scale) and "Would you have this type of anesthetic again?" (yes/no).

Data were analyzed using SPSS version 12. The incidences of postoperative anesthetic complications were expressed as a percentage of all anesthetics administered in the study population. Other descriptive univariate analyses were also obtained. Potential risk factors for postoperative complications were assessed using bivariate analysis and $\chi^{2}$-square test. A p-value $<0.05$ was accepted as statistically significant. After identifying significant variables by bivariate analysis, these were entered into multiple logistic regression models to examine each variable, while controlling for all other confounding factors.

\section{RESULTS}

Data were collected between June 2009 and September 2010. Five hundred and twenty-five (525) patients met the inclusion criteria and agreed to participate in the study, but 20 were excluded due to missing data. There were 374 females (74\%) and 131 males $(26 \%)$, with $58 \%$ being gynecological patients and $42 \%$ orthopedic. The mean age was $44.8 \pm 15.2$ years (range 16-88 years) and median 43 years. The majority were classified as either ASA I (55\%) or ASA II (38\%) (Table I). Most patients had their procedures performed under general anesthesia $(80 \%, n=404)$, and the remainder had either a neuraxial $(18 \%, n=91)$, or a peripheral nerve block $(1.5 \%$, $n=7)$. Two patients $(0.5 \%)$ had a combined technique (nerve block and general anesthesia). Intermediate grade procedures were seen most frequently $(83 \%)$ and most were between one and two hours duration (41\%) (Table I). The most common comorbidities seen were hypertension (26\%), diabetes mellitus (9.5\%), and asthma (7.5\%) (Table II). Approximately one third of patients (31\%) had one comorbidity and another $17 \%$ had multiple comorbidities.

Table I - Patient Characteristics

\begin{tabular}{ll}
\hline Characteristic & N\# (\%) \\
\hline Gender & $131(25.9)$ \\
Male & $374(74.1)$ \\
Female & \\
ASA status & $278(55.0)$ \\
ASA I & $192(38.0)$ \\
ASA II & $34(6.7)$ \\
ASA III & $1(0.2)$ \\
ASA IV & \\
Surgical Risk Grade & $16(3.2)$ \\
Minor & $419(82.8)$ \\
Intermediate & $70(13.8)$ \\
Major & \\
Length of Anesthesia & $58(11.5)$ \\
60 min. & $206(40.8)$ \\
$61-120$ min. & $145(28.7)$ \\
$121-180$ min. & $56(11.1)$ \\
$181-240$ min. & $40(7.9)$ \\
$>240$ & \\
Surgical Specialty & $293(58.0)$ \\
Gynecology & $212(42.0)$ \\
Orthopedics &
\end{tabular}


Table II - Incidence of Comorbidities

\begin{tabular}{lll}
\hline Comorbidities & Number & Percent \\
\hline Hypertension & 133 & 26.3 \\
Diabetes & 48 & 9.5 \\
Smoking habit & 43 & 8.5 \\
Asthma & 38 & 7.5 \\
Marijuana smoking & 26 & 5.1 \\
Heart disease & 10 & 2.0 \\
Renal disease & 9 & 1.8 \\
CVA & 9 & 1.8 \\
Sickle cell disease & 8 & 1.6 \\
Thyroid disease & 8 & 1.6 \\
Seizure disorder & 2 & 0.4 \\
COPD & 1 & 0.2 \\
\hline
\end{tabular}

CVA: Cerebrovascular accident; COPD; chronic obstructive pulmonary disease.

Table III - Intraoperative and Immediate Postoperative Complications

\begin{tabular}{lll}
\hline Complication & Number & Percent of all anesthetics \\
\hline Cardiovascular & 26 & $5.1 \%$ \\
Respiratory & 7 & $1.4 \%$ \\
Hemorrhage & 23 & $4.5 \%$ \\
Failed technique/ equipment & 22 & $4.3 \%$ \\
PONV & 5 & $1.0 \%$ \\
Other & 6 & $1.2 \%$ \\
\hline
\end{tabular}

PONV: postoperative nausea and vomiting.

\section{Intraoperative and post-anesthesia care unit complications}

A total of 77 patients (15\%) had a recorded complication either intraoperatively or in the immediate postoperative period. Five percent $(5 \%)$ of patients experienced cardiovascular complications, such as hypotension or hypertension and arrhythmias, and $4.5 \%$ experienced significant hemorrhage (blood loss $>10 \%$ of estimated blood volume). Only $1 \%$ of patients had nausea and vomiting in the immediate postoperative period (Table II). Eight patients had two complications and two had three complications. The recovery period had no significant incidents for most patients, with $96 \%(n=487)$ being discharged to the ward within four hours. Eighteen patients (4\%) were monitored in the postanesthesia care unit (PACU) for over six hours, usually for fluid or pain management, but were later discharged uneventfully to the ward. There were no ICU admissions, either immediately postoperatively or after being in the PACU.

\section{Postoperative complications}

A total of 419 (83\%) of all patients interviewed reported at least one complication postoperatively. The most frequent complaints were sore throat (44\%), nausea (30\%), vomiting (24\%), thrombophlebitis (20\%), and oral trauma (19\%). None of the patients who had general anesthesia reported awareness (Table III). The median VNRS for each complication ranged between 3 and 5 out of a total of 10 , and the mode was two to five, suggesting that most of the complications did not cause severe distress to patients (Table IV).
Table IV - Incidence of Postoperative Complications and Severity Scoring

\begin{tabular}{llll}
\hline Complication & Number (\%) & Median VNRS & Mode VNRS \\
\hline Sore throat & $223(44.2)$ & 3 & 2 \\
Nausea & $152(30.1)$ & 4 & 3 \\
Vomiting & $123(24.4)$ & 4 & 4 \\
Thrombophlebitis & $99(19.6)$ & 3 & 2 \\
Oral trauma & $97(19.2)$ & 3 & 2 \\
Back pain & $86(17.0)$ & 5 & 5 \\
Myalgia & $68(13.5)$ & 4 & 2 \\
Headaches & $54(10.7)$ & 3 & 2 \\
Micturition problems & $47(9.3)$ & 5 & 5 \\
Paresthesia & $46(9.1)$ & 5 & 3 \\
Motor deficit & $16(3.2)$ & 5 & 5 \\
Nightmares & $10(2.0)$ & 5.5 & 5 \\
Memory loss & $7(1.4)$ & 5 & 3 \\
Teeth trauma & $4(0.8)$ & 4 & 2 \\
Awareness & 0 & -- & -- \\
\hline
\end{tabular}

Table V - Complications versus Anesthetic Technique

\begin{tabular}{llllll}
\hline \multirow{2}{*}{ Complication } & \multicolumn{7}{l}{ Anesthetic Technique } & \\
& GA & RA & NB & Combined & p-value \\
\hline Headaches & 38 & 16 & - & - & $<0.001^{*}$ \\
Sore throat & 211 & 7 & 2 & 2 & $<0.001^{*}$ \\
Myalgia & 56 & 10 & 1 & - & 0.825 \\
Nausea & 128 & 22 & 1 & - & 0.490 \\
Vomiting & 98 & 23 & 1 & - & 0.573 \\
Oral trauma & 93 & 4 & - & - & $<0.001^{*}$ \\
Teeth trauma & 4 & - & - & - & - \\
Thrombophlebitis & 85 & 12 & 2 & - & 0.065 \\
Memory loss & 6 & 1 & - & - & 0.405 \\
Micturition problems & 36 & 10 & - & - & 0.952 \\
Nightmares & 9 & - & - & - & - \\
Back pain & 68 & 17 & - & - & 0.925 \\
Motor deficit & 12 & 2 & 1 & - & 0.163 \\
Paresthesia & 31 & 12 & 1 & 1 & $0.029^{*}$ \\
\hline
\end{tabular}

GA: General anesthesia; RA: Regional anesthesia; NB: Nerve block.

Significant correlations with postoperative complications included age, gender, surgical specialty, and anesthetic technique. The mean age of patients reporting complications was $43.4 \pm 14.2$ years compared to $51.8 \pm 19.3$ years in those without any complications. Eighty-seven percent $(87 \%)$ of patients aged less than 45 years reported at least one complication, compared to $78 \%$ of patients over 45 years $(p<0.001)$. Eighty-eight percent $(88 \%)$ of women reported complications versus $68 \%$ of men $(p<0.001)$. Gynecological patients were more likely to have complaints than orthopedic patients (89\% vs. $74 \%, p<0.001)$. Patients who received a general anesthetic had a higher percentage of complaints postoperatively than those who had a regional anesthetic $(87 \%$ vs. $65 \%$, respectively, $p<0.001$ ). Specific complaints also showed significant correlations; sore throat and oral trauma were associated with general anesthesia $(p<0.001)$ and headaches with regional anesthesia $(p<0.001)($ Table V). Logistic re- 
gression analysis was performed to identify the independent variables associated with postoperative complications. These included age less than 45 years (OR 2.22, 95\% $\mathrm{Cl}$ 1.34-3.69, $\mathrm{p}=0.002$ ), and female gender (OR $3.64,95 \% \mathrm{Cl} 2.14-6.20$, $\mathrm{p}<0.001)$. Controlling for gender resulted in surgical specialty no longer being significant, and anesthetic technique also lost significance after controlling for age.

The risk of developing minor postoperative complications did not correlate with: ASA status $(p=0.069)$, one comorbidity $(p=0.479)$, multiple comorbidities $(p=0.052)$, complications intraoperatively or in the PACU $(p=1.000)$, procedure risk grade $(p=0.330)$ or duration $(p=0.202)$. Of note, the presence of one comorbidity $(p=0.032)$ and multiple comorbidities $(p=0.01)$ were significantly correlated with intraoperative and PACU complications.

\section{Anesthetic experience rating}

Half of the patients interviewed (51\%) regarded their anesthetic experience as excellent, and another $22 \%$ thought it was very good. Ten patients $(2 \%)$ described their experience as 'poor', reporting on average 2.5 complications. Eight (8) of these underwent a general anesthetic. Of these 10 patients, eight also had at least one complication with a VNRS of 5 or greater, and three had scores of 10 (one each for sore throat, vomiting, and nightmares). The majority (92\%) indicated that they would opt to have the same anesthetic technique again in the event of another surgical procedure.

\section{DISCUSSION}

Minor morbidity, such as postoperative nausea and vomiting (PONV), sore throat, and headaches may have a significant impact on recovery from anesthesia, with decreased function and slower resumption of normal daily activities post discharge ${ }^{2}$. The frequency of postoperative complications varies widely in the literature. An analysis of postoperative complications seen in a large teaching hospital between 1979 and 1983 including over 60,000 patients found incidences of $0.04 \%$ for major and $9.4 \%$ for minor postoperative complications ${ }^{5}$. However, a study which looked specifically at minor postoperative complications after general anesthesia in 4,173 patients reported an incidence of $41 \%{ }^{8}$. The difference in findings in these two papers may reflect a difference in methodology. The former documented symptomatology voluntarily offered by the patient, while the latter questioned patients using a predetermined list of outcomes/complications. Our study, using a similar method, also showed a high incidence of minor complications (83\%).

A comprehensive review of articles published between 1966 and 2003 investigating peri-operative risk and complications associated with anesthesia found a wide range of incidences for specific minor complications ${ }^{2}$. We found sore throat to be a relatively common though seldom severe complaint, with an incidence of $44 \%$. A reported range of $14 \%$ to $64 \%$ following tracheal intubation ${ }^{2}$ suggests that improvement such as less aggressive suctioning could be made in our technique to lower our incidence. Our incidences of nausea and vomiting were $30 \%$ and $24 \%$, respectively, which again fell within the reported range of between $20 \%$ and $30 \%{ }^{2}$. The only other study from Jamaica that assessed PONV outside the recovery room period was in laparoscopic and open cholecystectomy patients who were assessed at 24 hours postoperatively ${ }^{9}$. Their overall incidence of $28.7 \%$ is in keeping with our findings.

Our incidence of overall myalgia was $13.5 \%$, which based on the findings of other investigators (1.5 to $89 \%$ ) was not unusual $^{2}$, but we did not document the use of suxamethonium or intramuscular injections, both of which may have impact on myalgia. However, it is the common practice at our institution to avoid suxamethonium for elective patients, barring a specific indication. Intramuscular injections on the other hand are almost universally used to deliver analgesics because this method is cheap and simple. This practice may need to be reviewed.

Our combined incidence of oral and teeth trauma was $20 \%$, though damage to teeth only accounted for $0.8 \%$. This was high compared to a reported incidence of $7 \%$ for all types of oral trauma (from soft tissue laceration to tooth fracture or avulsion) in intubated patients ${ }^{2}$. This high figure could be related to the fact that our institution is a teaching hospital, with training of undergraduate and postgraduate students who are less skilled in airway instrumentation to avoid oral trauma. This is an area for further investigation to determine the cause, develop and implement changes designed to reduce the occurrence of complications. We also documented a $17 \%$ incidence of back pain, which was higher than observed in other studies ${ }^{5,8}$. We did not find a significant association with spinal anesthesia ( $p=0.9250$, Table $V$ ) and were unable to comment on any influence of patient positioning (e.g., lithotomy), as we did not collect this information.

We found no correlation between the physical status ASA score and frequency of minor complaints. This could have been due to the low number of ASA III and IV patients (6\%), making it difficult to make comparisons. In addition, work by Lee et al. ${ }^{10}$ suggests that there is an inconsistent correlation of ASA scores with minor postoperative morbidity, as opposed to a stronger correlation with major complications and mortality.

It has been shown in several studies that women have a greater risk of minor postoperative complications, such as nausea and vomiting, headache and backache ${ }^{4,8}$. We also demonstrated this correlation, with a greater percentage of females reporting complaints ( $88 \%$ vs $68 \%$ ). One possible explanation proposed is that it is more socially acceptable for women to express their discomfort, while males tend to underreport complications. Hormonal differences seem unlikely, as no difference has been demonstrated between pre and postmenopausal women ${ }^{8}$.

In this study, younger patients were also more likely to have complaints than older patients, and the difference was most marked in patients less than 45 years. Our observations 
may reflect greater stoicism in older patients ${ }^{11,12}$ who may also have had previous experience with anesthesia, and thus may be less likely to report minor problems even when interviewed. This is supported by studies of patient satisfaction post-anesthesia that report higher satisfaction scores in older patients. This difference, however, was seen in patients over 65 years of age ${ }^{4}$.

It is interesting that despite a high overall incidence of complaints, general satisfaction with anesthesia was high. We are unable to identify any reasons for this apparent discrepancy in our findings. Satisfaction is "the balance between what is expected and the perception of what was received" ${ }^{13}$. It may be that our patients expected some degree of discomfort postoperatively, and hence were less likely to give a poor score for an experience that was within their expectations. Another possibility could be an attempt to please the interviewer and create a favorable impression.

\section{Limitations}

Patient interviews have the potential for either under- or overreporting complications due to recall bias ${ }^{14}$. The patient satisfaction questions did not specify particular aspects of care, but were general and non-specific. Single global ratings may be insensitive to actual deficiencies in care. The lack of variability in ASA, risk grade, and length of anesthesia amongst the population studied may have interfered with the observa- tion of no correlation of these variables with complications. Additionally, this study may have not been adequately powered to detect low frequency complications, such as awareness (reported incidence of $<0.3 \%$ ), which may account for it not being observed.

\section{CONCLUSION}

This study showed a high incidence of minor postoperative complication (83\%), but low reported severity of symptoms and a high overall satisfaction rate with anesthesia. More complications were seen in younger and female patients, and sore throat, nausea, vomiting, and oral trauma were the most frequent. Special attention should be paid to reduce these complications through a more meticulous anesthetic technique. Our objective with each anesthetic should be to provide a safe and comfortable experience for the patient.

\section{ACKNOWLEDGEMENTS}

The authors would like to thank the nurses of the Research Office, Department of Surgery, Radiology, Anesthesia and Intensive Care, for their assistance in data collection and entry. We would also like to thank Professor R. Carpenter for his invaluable advice throughout this project. 


\section{REFERÊNCIAS/REFERENCES}

1. Kehlet $H$, Dahl JB - Anaesthesia, surgery, and challenges in postoperative recovery. Lancet, 2003;362:1921-1928.

2. Jenkins $K$, Baker $A B$ - Consent and Anesthetic risk. Anaesthesia, 2003;58:962-984.

3. Domino KB, Posner KL, Caplan RA, Cheney FW - Awareness during anesthesia: a closed claims analysis. Anesthesiology, 1999;90:10531061.

4. Myles PS, Williams DL, Hendrata M, Anderson H, Weeks AM - Patient satisfaction after anaesthesia and surgery: results of a prospective survey of 10,811 patients. Br J Anaesth, 2000;84:6-10.

5. Cohen MM, Duncan PG, Pope WD, Wolkenstein C - A survey of 112,000 Anesthetics at one teaching hospital (1975-83). Can Anaesth Soc J, 1986;33:22-31.

6. Tennant IA, Augier R, Crawford-Sykes A, Hambleton IR, Tha M, Harding $\mathrm{H}-$ Anesthetic morbidity at the University Hospital of the West Indies. West Indian Med J, 2009;58:452-459.

7. Cohen MM, Duncan PG, Tweed WA et al. - The Canadian four-centre study of Anesthetic outcomes: I. Description of methods and populations. Can J Anaesth, 1992;39:420-429.

8. Myles PS, Hunt JO, Moloney JT - Postoperative minor complications. Comparison between men and women. Anaesthesia, 1997;52:300306.

9. East JM, Mitchell DIG - Postoperative nausea and vomiting in laparoscopic versus open cholecystectomy at two major hospitals in Jamaica. West Ind Med J, 2009;58:130-137.

10. Lee A, Lum ME - Measuring Anesthetic outcomes. Anaesth Intensive Care, 1996;24:685-693.

11. Layzell $\mathrm{M}$ - Exploring pain management in older people with hip fracture. Nursing Times, 2009;105:20-23.

12. Parker $\mathrm{A}$ - Care of the older perioperative patient. $\mathrm{Br} \mathrm{J}$ of Anesthetic and Recovery Nursing, 2002;8:21-27.

13. Capuzzo M, Gilli G, Paparella $L$ et al. - Factors predictive of patient satisfaction with anesthesia. Anesth Analg, 2007;105:435-442.

14. Hennekens $\mathrm{CH}$, Buring JE - Epidemiology in medicine. Philadelphia: Lippincott Williams and Wilkins; 1987, p. 274. 